\title{
The Formation of Social Network Assortativity: A Cultural Trait-Matching Mechanism
}

\author{
Wei Wang $\left(D,{ }^{1}\right.$ Xiaoming Sun $\mathbb{D}^{2},{ }^{2}$ Yalan Wang, ${ }^{2}$ and Wentian $\mathrm{Cui}^{3}$ \\ ${ }^{1}$ Business School, Zhengzhou University, Zhengzhou 450001, China \\ ${ }^{2}$ School of Management, Xi'an University of Architecture and Technology, Xi'an 710055, China \\ ${ }^{3}$ School of Management, Xi'an Jiaotong University, Xi'an 710049, China \\ Correspondence should be addressed to Xiaoming Sun; sun413@xauat.edu.cn
}

Received 17 February 2020; Revised 4 November 2020; Accepted 28 November 2020; Published 7 December 2020

Academic Editor: Klaus Jaffe

Copyright (C) 2020 Wei Wang et al. This is an open access article distributed under the Creative Commons Attribution License, which permits unrestricted use, distribution, and reproduction in any medium, provided the original work is properly cited.

The preferential attachment mechanism that forms scale-free network cannot display assortativity, i.e., the degree of one node is positively correlated with that of their neighbors in the network. Given the attributes of network nodes, a cultural trait-matching mechanism is further introduced in this paper. Both theoretical analysis and simulation results indicate that the higher selection probability of such mechanism, the more obvious the assortativity is shown in networks. Further, the degree of nodes presents a positive logarithm correlation with that of adjacent ones. Finally, this study discusses the theoretical and practical significances of the introduction of such a cultural trait-matching mechanism.

\section{Introduction}

The concept of assortativity was introduced by Newman in 2002 [1]. Note that Newman [1] put forward assortativity in the context of social networks rather, some scholars investigated assortative mating, characterized as "self-seeking like," and assortation in broader contexts beyond networks (see Schwartz's work [2] for a review), such as mate selection strategies, evolution of sex, choice of pets, genetic algorithms, and emergence and maintenance of social cooperation. In this study, we limit the implications of assortativity to social networks [1]. Namely, the high-degree nodes in social networks are, on average, connected to other nodes with high degree, and low degree nodes are, on average, connected to other nodes with low degree $[1,3,4]$. In short, similarity breeds connection [5]. Henceforth, assortative behaviors have been widely quantified and mimicked in a variety of real-world networks, e.g., Internet, World-Wide Web, Facebook, physics coauthorship, mathematics coauthorship, company directors, nand eutral network (see [6] for a review). Meanwhile, scale-free networks, given by Barabasi and Albert $[7,8]$, are formed by the preferential attachment mechanism. However, Newman proved that the assortativity coefficient of a network formed by preferential attachment mechanism tended to be zero when the network reached a very large scale [1]. Such a phenomenon is not consistent with the properties of real-life social networks. Therefore, a lot of scholars have investigated the issues as to the formation and characteristics of assortativity [5, 9-19]. Yet, our understanding of the role of cultural characteristics in the formation of social network assortativity is fairly limited.

With respect to the formation of assortativity, Newman and Park [9] introduced a network model based on the formation mechanism of community networks. Using this model, they proved that since a social network could be divided into communities, community structure in turn resulted in positive correlation of node degree. In contrast, nonsocial networks did not usually present such structures. They therefore argued that the assortativity of social networks, which could not be found in nonsocial networks, may be attributable to community structure. Catanzaroa et al. $[10,11]$ established a mathematical model to reproduce the assortativity of collaborative networks between scientists specializing in condensed matter physics, wherein the networks were regarded as being derived from two 
mechanisms. One is the preferential attachment mechanism of the BA model, and the other is the mechanism allowing for the connection probability being determined by the similarity of the degrees of two nodes in embordering two unconnected nodes. Guo et al. [12] developed a combined (scale-free small-world) network with adjustable assortativity coefficients, which overcame the defect of the assortativity of a network tending to zero when the network scale became large. Vázquez [14] asserted that the local-world connection mechanism was important in the formation of degree correlation. Xulvi-Brunet and Sokolov [15] proposed an algorithm that could produce arbitrary sized assortativity coefficients to explore the assortativity in networks. They further applied this algorithm to the BA model and concluded that the network assortativity positively correlated with the shortest network path.

Rather than the formation of assortativity, some scholars explored the characteristics of assortativity. For example, existing research of assortativity mainly focused on one dimension of attributes at a time, Block and Grund [16], instead, investigated multidimensional assortativity (also called as homophily in the study) in the context of network dynamics. They found that main effects for assortativity on various dimensions were positive, while the interaction of assortativity effects was negative. Peel et al. [17] argued that the distribution of assortativity in many real-world networks was skewed, overdispersed, and multimodal. Similarly, Piraveenan et al. [18] introduced a measure of local assortativity and found the various distribution of local assortativity in biological, social, and technological networks. Further, based on scalar assortativity, the extension of assortativity proposed by Newman [3], Piraveenan et al. [19] found scalar assortativity carried information about network dynamics.

Recent literature reviewed above, however, is mainly from the perspective of physical evolution mechanisms and physical description, and limited attention has been paid to the role of social attributes of nodes in network assortativity. Notably, a recent study of Cantwell and Newman [4] indicated that assortativity captured only the average mixing behavior of nodes, neglecting individual difference in networks. They introduced a generative stochastic model to unveil the individual variation in mixing patterns, further argued that traditional average measures of assortative mixing offer an incomplete description of network structure. In line with this argument, Altenburger and Ugander [20] concentrated on the variability of assortativity. In the study of Facebook membership, they found that some individuals with extreme preferences for a particular attribute possibly unrelated to their own attribute, which was called as monophily. Therefore, the individual attributes of nodes should be emphasized in assortativity research. Actually, assortativity have been applied to some social attributes of nodes, including race [21], language [22], age [23], nationality [24], and social status [25]. As an example, employing sociological theory, Postmes et al. [25] pointed out that, although ordinary people expected to establish connections with celebrities or industry elites, these celebrities or industry elites preferred to communicate or cooperate with those of perceived equal social standing. Such phenomenon resulted in the positive correlation of node degree. Hence, positive degree correlation could only possibly be observed in such competitive scenarios. In contrast, online social networks, which broke competitive and social class, showed disassortativity [25]. Furthermore, Postmes et al. [25] speculated that positive correlation also depended on the irreplaceability of two collaborative parties, i.e., the research subject and knowledge background of researchers or the performing style of entertainers. Meanwhile, Liu et al. [13] designed a weighted network model by integrating the mutual selection mechanism between nodes and the self-learning mechanism. They proposed that, in the case of high probability of self-learning and low value of mutual selection, the assortativity of social networks emerged.

On the other hand, empirical research also suggested that a social network was composed of individuals with various cultural characteristics. These individuals built relationships according to own cultural characteristics. The relationships were thus developed into a network group with different structures and characteristics $[5,26]$.

Above two lines of arguments indicate that relationships between social individuals are not established completely in accordance with the preferential attachment mechanism. In other words, social individuals are not always prone to select nodes with higher connection degrees. Instead, they refer to the mechanisms that including more social characteristic, for example, cultural characteristics [27]. In sum, this study constructs a social network using cultural characteristic matching as its connection mechanism. Moreover, it explains the assortativity of a social network from the perspective of social attributes.

\section{A New Network Model with Cultural Trait- Matching Mechanism}

2.1. Cultural Traits and Features. Culture traits and features are described using the social individual culture characterizations proposed by Axelrod [27].

(1) Cultural traits refer to the society's cultural symbols as selected by individuals. For example, social individuals can select one language (e.g., Chinese, English, and Korean) as their communication tool; each language is thus an independent cultural trait.

(2) Cultural features are abstract concepts of different cultural traits of individuals. For example, Chinese, English, and Korean can be abstractly defined as languages; Buddhism, Taoism, and Christianity can be defined as religious beliefs [28]. In turn, language is a cultural feature, and individuals can choose Chinese, English, or Korean as their own cultural traits. As noted by Axelrod [27], “... .it describes a culture as a list of features or dimensions of culture. For each feature there is a set of traits, which are the alternative values the feature may have." 
2.2. Model Assumption. Social individuals are heterogeneous $[29,30]$. Merely from the viewpoint of culture, each social individual has his/her different cultural traits. These specific cultural traits lead to the differences between social individuals [27].

Connections of social individuals are built by two selection mechanisms. One is the preferential attachment mechanism [31], in which individuals mainly focus on the degree of nodes being connected. To be concrete, they are more concerned about the influences of target connection nodes, rather than whether or not the cultural traits of the target node match theirs. This phenomenon is widespread. In many cases, for instance, the connections between social individuals are a result of blind follow or demand on the influences of the other party, such as power or reputation. The other is the cultural trait-matching mechanism. With this mechanism, individuals are more likely to connect with nodes bearing higher matching degree with their cultural features $[5,25,27]$. This phenomenon is also common in practice. For example, amongst three people, two people who share a love of calligraphy are more likely to establish a connection and further become friends.

2.3. Network Formation. Employing Axelrod's cultural diffusion model [27], each individual or agent $i$ has its cultural features defined as a vector of $F$; each feature takes its integer values from a range of $q$ possible cultural traits [28]. Thus, $q^{F}$ different cultural trait vectors are obtained $[32,33]$. To be concrete, the formation mode of cultural features by individual $i$ is represented as vector $c_{i}=\left(\alpha_{i 1}, \alpha_{i 2}, \ldots, \alpha_{i F}\right)$, where $\quad \alpha_{i, f} \in C_{f}, i=1,2, \ldots, N$; $f=1,2, \ldots, F ; C_{f}=\{1,2,3, \ldots, q-1, q\},\left|C_{f}\right|=q[33]$. The formation steps of networks are as follows:

(1) Initialize a fully connected network with $m_{0}$ nodes, and assign a cultural feature vector for each node.

(2) At each time step $t$, a new node is added and connected with nodes available in the network. This new node has a cultural feature vector $c_{t}$ and $m$ edges $\left(m \leq m_{0}\right)[7,8]$.

(3) The new node selects a preferential attachment mechanism with the probability $p$ and adopts a cultural trait-matching connection mechanism according to probability $1-p$. If the new node chooses a preferential attachment mechanism, it is connected to the existing node $i$ with the probability in equation (1), where $j$ denotes arbitrary node in the network except the newly introduced one [31]. Besides, if the new node chooses a cultural trait-matching connection mechanism, it is necessary to firstly calculate the cultural trait-matching degree between the new node and the existing one $i$ by using equation (2). Further, the new node is connected to the nodes in the network with the probability in equation (3):

$$
\begin{aligned}
\prod\left(k_{i}\right) & =\frac{k_{i}}{\sum_{j} k_{j}}, \\
M_{t i} & =\sum_{f=1}^{F} \lambda_{\alpha_{t f}, \alpha_{i f},} \\
\lambda_{\alpha_{t f}, \alpha_{i f}} & = \begin{cases}1, & \text { if } \alpha_{t f}=\alpha_{i f}, \\
0, & \text { if } \alpha_{t f} \neq \alpha_{i f},\end{cases} \\
P\left(M_{t i}\right) & =\frac{M_{t i}}{\sum_{j} M_{t j} .}
\end{aligned}
$$

(4) Repeat steps (2) and (3) until the network scale reaches $N$.

\section{Theoretical Analysis}

3.1. Degree Distribution. At each time step, a node with a cultural feature vector enters the network. Assuming that the elements in $C_{f}$ are selected with equal probability $1 / q$, not only within a cultural feature $C_{f}$ but also between across different cultural feature sets [32]. As a consequence, a selection cycle emerges, i.e., $T=q^{F}$, it can be considered that at each time $T$, and all cultural feature vectors are uniformly selected once and enter the network along with the associated nodes $[32,33]$. Therefore, at time $t$, when a new node enters the network, the cultural trait-matching degree between the original node and this new one develops from completely mismatching, matching only one culture feature, to full matching. The matching degree can be numerically represented as $[0,(1 / F),(2 / F), \ldots, 1][27,28]$. Accordingly, at time $t$, the sum of matching degree between original nodes and the new node is $S=\left(\left(\left(m_{0}+t\right)\right) / q^{F}\right) \times s_{0}$, where $s_{0}$ can be expressed by equation (4) [28]. It refers to the theoretical sum of matching degree in one selection cycle. The matching degree of the new node with original nodes in the network is set as a random, time independent variable:

$$
\begin{aligned}
s_{0}= & C_{F}^{0}(q-1)^{F} \times 0+C_{F}^{1}(q-1)^{F-1} \times \frac{1}{F} \\
& +\cdots+C_{F}^{F}(q-1)^{F-F} \times 1 .
\end{aligned}
$$

According to mean field theory and the $k$ continuum hypothesis $[7,8]$, the dynamical equation of the degree $k_{i}$ of node $i$ can be obtained:

$$
\frac{\partial k_{i}}{\partial t}=A \prod\left(k_{i}\right), \prod\left(k_{i}\right)=\prod_{1}\left(k_{i}\right)+\prod_{2}\left(k_{i}\right)
$$

where the value of $A$ is $m=2 . \prod_{1}\left(k_{i}\right)$ and $\prod_{2}\left(k_{i}\right)$ are the probabilities of preferential attachment and culture trait matching, respectively:

$$
\frac{\partial k_{i}}{\partial t}=m\left[p \frac{k_{i}}{2 m t+m_{0}}+(1-p) \frac{L q^{F}}{\left(m_{0}+t\right) s_{0}}\right]
$$


where $L$ is the matching degree of new added node with its arbitrary original node. The expected value of $L$ is $E(L)=$ $\left(\left(C_{F}^{0}(q-1)^{F} / q^{F}\right) \times 0\right)+\left(\left(C_{F}^{1}(q-1)^{F-1} / q^{F}\right) \times(1 / F)\right)+\cdots+$ $\left(\left(C_{F}^{F}(q-1)^{F-F} / q^{F}\right) \times 1\right)$, thus

$$
\frac{\partial k_{i}}{\partial t} \approx p \frac{k_{i}}{2 t}+(1-p) \frac{m L q^{F}}{t s_{0}} .
$$

Let $G=\left(L q^{F} / s_{0}\right)$, the degree of node $i$ at time $t$ can be calculated by equation (7):

$$
k_{i}(t)=\left[m+\frac{2 m(1-p) G}{p}\right]\left(\frac{t}{t_{i}}\right)^{(p / 2)}-\frac{2 m(1-p) G}{p} .
$$

According to the definition of degree distribution,

$$
P(k, t)=\frac{\partial P\left\{k_{i}(t)<k\right\}}{\partial k} .
$$

Substituting equation (8) into equation (9), we obtain

$$
P(k, t)=\frac{2}{p m+2 m(1-p) G}\left[\frac{2 m(1-p) G+p k}{p m+2 m(1-p) G}\right]^{(-(2+P) / P)} .
$$

(1) When $p=1$, such a model completely transforms into the BA model, in which the power law index of $k$ is $\gamma(p)=((2+p) / p)=3$.

(2) When $p=0$, $V$,

Let $[((2 m(1-p) G+k p) /(m p+2 m(1-p) G))]^{-(2 / p)}=$

$$
\begin{aligned}
\lim _{p \longrightarrow i} \ln V & =-\lim _{p \longrightarrow i}\left\{\frac{\ln [((2 m(1-p) G+k p) /(p m+2 m(1-p) G))]^{2}}{p}\right\}_{p} \\
& =\lim _{\text {pim }} \frac{[((m p+2 m(1-p) G) /(2 m(1-p) G+k p))]^{2}\left\{[(2 m(1-p) G+k p) /(m p+2 m(1-p) G)]^{2}\right\}_{p}}{1} .
\end{aligned}
$$

By solving equation (12), we can find

$$
V=\frac{m-k}{2 m^{2} G^{2}} .
$$

Hence, when $p=0$, the degree distribution is

$$
P(k, t)_{p=0}=\frac{1}{m G} e^{-\left(k / 2 m^{2} G^{2}\right)+\left(1 / 2 m G^{2}\right)} .
$$

3.2. Degree Correlation. Let $\delta=(p / 2)$ and $Z_{s}(t)$ is the sum of the degrees of adjacent nodes of node $s$. For arbitrary node $j$ in adjacent node set $\vartheta(s)$ of node $s$, there is

$$
Z_{s}(t)=\sum_{j \in \vartheta(s)} k_{j}(t)
$$

Thus, the mean degree of adjacent nodes of node $s$ is

$$
k_{n n}(s)=\frac{Z_{s}(t)}{k_{s}(t)} .
$$

The rate equation of $Z_{s}(t)$ [34] is

$$
\frac{d Z_{s}(t)}{\mathrm{d} t}=m\left[m \prod_{s}(\{k\}, t)\right]+m \sum_{j \in 9(s)} \prod_{j}(\{k\}, t) .
$$

In equations (15), (16), and (17), $\{k\}=\left\{k_{1}(t), k_{2}(t), \ldots, k_{t-1}(t)\right\}$. The first section on righthand side of equation (17) denotes that, when new node $t$ is directly connected to node $s$, the degree of adjacent nodes of node $s$ increases by $m$ units. The second section on the righthand side of equation (17) delineates that, when new node $t$ is directly connected with the adjacent nodes of node $s$, the degree of adjacent nodes of node $s$ increases by one unit. Therefore, to solve the rate equation, the boundary conditions should be obtained, i.e., the sum of degrees for all the adjacent nodes of node $s$ at time $s$ :

$$
Z_{s}(s)=m \sum_{j=1}^{s} \prod_{j}(\{k\}, s)\left[k_{j}(s)+1\right] .
$$

This implies that, at time $s$, new node $s$ is connected to an available node with degree of $k_{j}(s)$ in the network. According to this analysis, the probability of node $s$ being connected to the available node is

$$
\prod_{s}(\{k\}, t)=p \frac{k_{i}}{2 m t+m_{0}}+(1-p) \frac{L q^{F}}{\left(m_{0}+t\right) s_{0}} .
$$

Substituting equation (19) into the rate equation (17), we obtain 


$$
\begin{aligned}
\frac{d Z_{s}(t)}{\mathrm{d} t}= & m^{2}\left[\frac{p k_{s}(t)}{2 m t+m_{0}}+(1-p) \frac{G}{m_{0}+t}\right] \\
& +m \sum_{j \in \vartheta(s)}\left[\frac{p k_{j}(t)}{2 m t+m_{0}}+(1-p) \frac{G}{m_{0}+t}\right] \\
\approx & \delta \frac{(m+\kappa) k_{s}(t)+\kappa m}{t}+\delta \frac{Z_{s}(t)}{t},
\end{aligned}
$$

where $\kappa=(2 m G(1-p) / p)$. By solving the rate equation, the general solution of $Z_{s}(t)$ becomes

$$
Z_{s}(t)=B_{0}(s) t^{\delta}+\beta(m+\kappa)^{2}\left(\frac{t}{s}\right)^{\delta} \ln t+\kappa^{2} .
$$

where $B_{0}$ is given by boundary conditions:

$$
\begin{aligned}
Z_{s}(s) & =m \sum_{j=1}^{s}\left[p \frac{k_{j}(s)}{\left(2 m+m_{0}\right) t}+(1-p) \frac{G}{m_{0}+t}\right]\left[k_{j}(s)+1\right] \\
& =\delta \kappa+2 m \delta(\kappa+1)+\frac{\delta}{s} \sum_{j=1}^{s} k_{j}^{2}(s) .
\end{aligned}
$$

Substituting $k_{j}(s)=(m+\kappa)(s / j)^{(p / 2)}-\kappa$ into equation (22) gives

$$
Z_{s}(s)=m(1-\kappa)+\delta(m+\kappa)^{2} s^{2 \delta-1} \sum_{j=1}^{s} j^{-2 \delta} .
$$

(1) When $p=1, \kappa=0$, and $\gamma=3$ [31], the result indicates that the network evolved from the model is a scale-free network. Ignoring the fact that the limit is zero when $t$ and $s$ are large, as independent variables, since $\sum_{j=1}^{s} j^{-1} \simeq \ln s, Z_{s}(s) \simeq\left(m^{2} / 2\right) \ln s$ is obtained,

$$
Z_{s}(t) \simeq \frac{m^{2}}{2} \sqrt{\frac{t}{s}} \ln s .
$$

Under the limiting condition that when $k$ and $N$ are large, $s$ is replaced by the function of $s$ in $k$, we get $s=t k^{-(1 / \beta)}(m+a)^{(1 / \beta)}$. Using the final scale $N$ of the network to replace $t$, when $t=N$, according to equation (16), we find that

$$
k_{n n}(k, N) \simeq \frac{m}{2} \ln N .
$$

Obviously, the degree correlation of two nodes in a scale-free network is related with the scale of network but independent of the degrees of the nodes. Thus, the assortativity coefficient of a scale-free network is zero [1].

(2) When $0<p<1$, namely, $\kappa>0, \gamma>3$, we get $\sum_{j=1}^{s} j^{-2 \delta} \simeq\left(s^{1-2 \delta} /(1-2 \delta)\right)$, thus

$$
\begin{aligned}
Z_{s}(t) \simeq \delta & (m+\kappa)^{2}\left(\begin{array}{l}
t \\
s
\end{array}\right)^{\delta} \ln \left(\begin{array}{l}
t \\
s
\end{array}\right) \\
& +\left[m(1-\kappa)+\frac{\delta(m+\kappa)^{2}}{1-2 \delta}-\kappa^{2}\right]\left(\begin{array}{l}
t \\
s
\end{array}\right)^{\delta} .
\end{aligned}
$$

Therefore, the degree correlation function of a given node is

$$
k_{n n}(k, N) \simeq[m+\kappa] \ln \frac{k}{m+\kappa} .
$$

Equation (27) shows that, under these conditions, as for any node, the mean degree function of its adjacent nodes presents a positive logarithmic correlation with node degree but is unrelated to network scale. Therefore, the network displays assortativity.

\section{Simulation Analysis}

The initial network in this paper includes 10 nodes with fully connected. Besides, at each time step, we induce two edges into the network. With MATLAB ${ }^{\circledR}$ and Monte Carlo simulation method, we use the algorithm of roulette wheel selection to set the mode of node connection. Further, we run the program under each condition for 10 times and get the final results from averaging the values at each point.

4.1. Degree Distribution in Social Networks. Based on equation (8), we depict the variation characteristics of degree distribution under $N=5000$ and different parameters in Figure 1 , where $p=1, p=0$, and $p=0.4$, respectively, describe the network degree distribution generated totally by the preferential attachment mechanism of BA network, wholly by the cultural trait-matching connection mechanism, and by the combination of above two mechanisms with a probability of 0.4 and 0.6 . According to the characteristics of degree distribution, we can see clearly that, with the influence of cultural trait matching, the social network generated shows a wide degree distribution and follows a power law.

4.2. Influences of Connection Mechanism pon the Assortativity of Social Networks. Figure 2 describes the variation characteristics of assortativity coefficient $\gamma$ under different network scales and selection probabilities of preferential attachment when $q=5$ and $F=6$. It can be found that, under the same network scale, the larger $p$ is, the smaller $\gamma$ is; under the same preferential attachment mechanism selection probability $p, \gamma$ decreases with increasing network scale $N$. However, when $p$ is small, variations in $\gamma$ diminish.

The assortativity coefficient $\gamma$ is calculated by equation (28) $[1,12]$, which is employed to analyze the causes of variation in Figure 2, where $j_{i}$ and $k_{i}$ represent the degrees of two nodes connected by the $i$ th edge and $M$ refers to the number of edges in the network: 


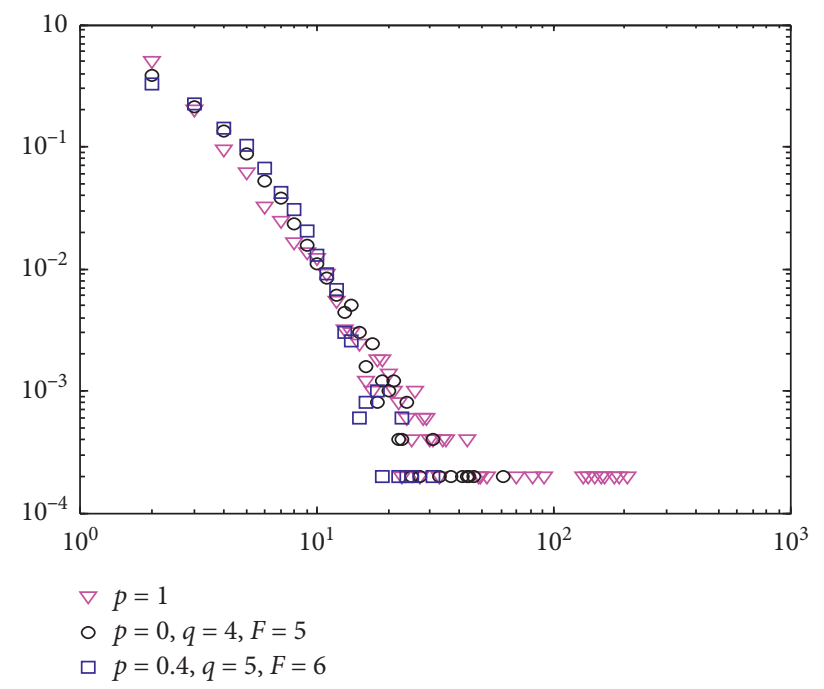

FIgURE 1: Degree distribution for different probabilities of the connection mechanism.

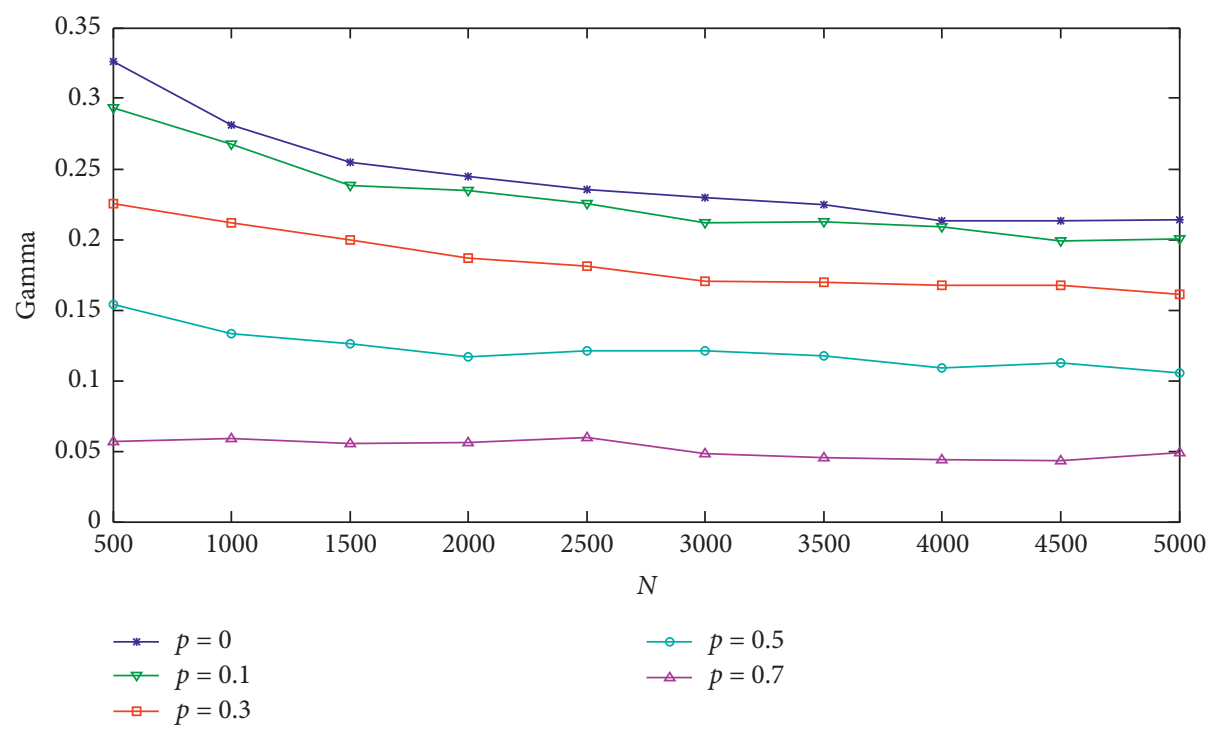

Figure 2: Assortativity coefficients for different network scales and probabilities of connection mechanism.

$$
\gamma=\frac{M^{-1} \sum_{i} j_{i} k_{i}-\left[M^{-1} \sum_{i}(1 / 2)\left(j_{i}+k_{i}\right)\right]^{2}}{M^{-1} \sum_{i}(1 / 2)\left(j_{i}^{2}+k_{i}^{2}\right)-\left[M^{-1} \sum_{i}(1 / 2)\left(j_{i}+k_{i}\right)\right]^{2}} .
$$

Given equation (28), both the numerator and the denominator minus one same term, the network assortativity coefficient is thus determined by the relationship of $j_{i} k_{i}$ and $(1 / 2)\left(j_{i}^{2}+k_{i}^{2}\right)$, where $j_{i}^{2}+k_{i}^{2} \geq 2 j_{i} k_{i}$. Moreover, when and only when $j_{i}=k_{i}, j_{i}^{2}+k_{i}^{2}=2 j_{i} k_{i}$. Therefore, it can be found that the closer the degrees of two nodes connected by one edge, the larger the assortativity coefficient of the network, and vice versa.

Besides, under the preferential attachment mechanism, new nodes are more likely to connect with nodes, which have higher degree in the network. Such phenomenon is called as Matthew effect (i.e., the rich get richer). Further, with the increasing of network size, this effect is enlarged. Therefore, a small number of hubs with very large degrees are generated. Given that a few hubs are connected with a large amount of nodes bearing very low degrees, the network's assortativity coefficient is thus very low. However, when the matching mechanism of cultural traits is introduced, the formation of hubs by preferential attachment mechanism is weakened. Moreover, due to the heterogeneity of cultural traits, node selection is no longer limited to the influence of node degree. Thus, the degrees of nodes are relatively uniform and the network's assortativity coefficient is higher.

4.3. Simulation of Degree Correlation of Nodes. Figure 3 shows the comparison of simulated and theoretical results of degree correlation under different parameters when $N=5000$ ( $L$ is approximated using its expected value). It can 

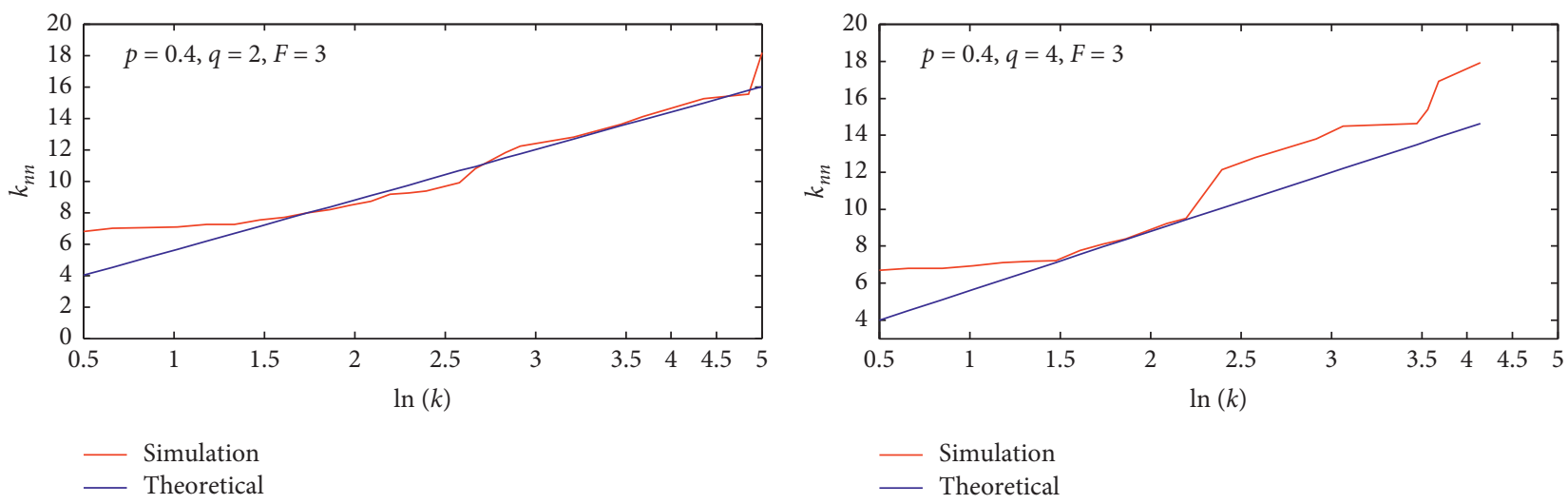

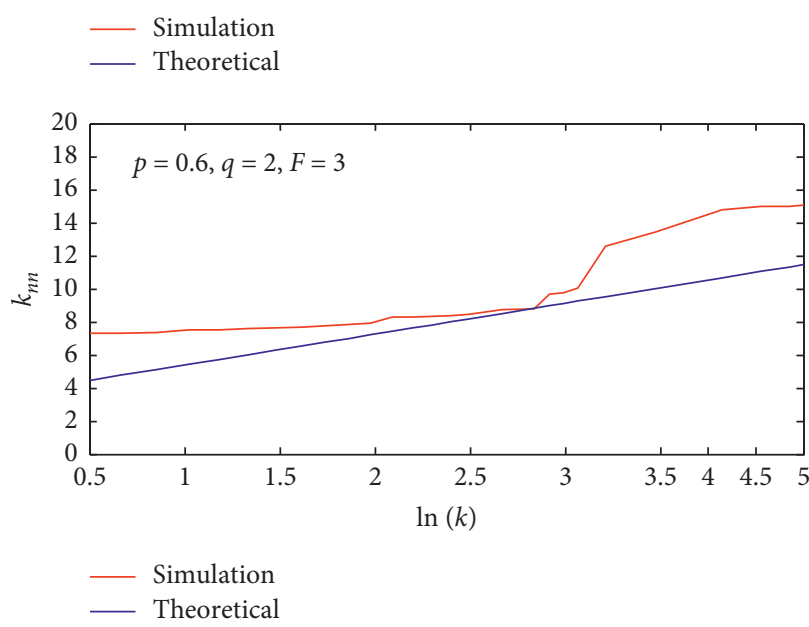

(a)

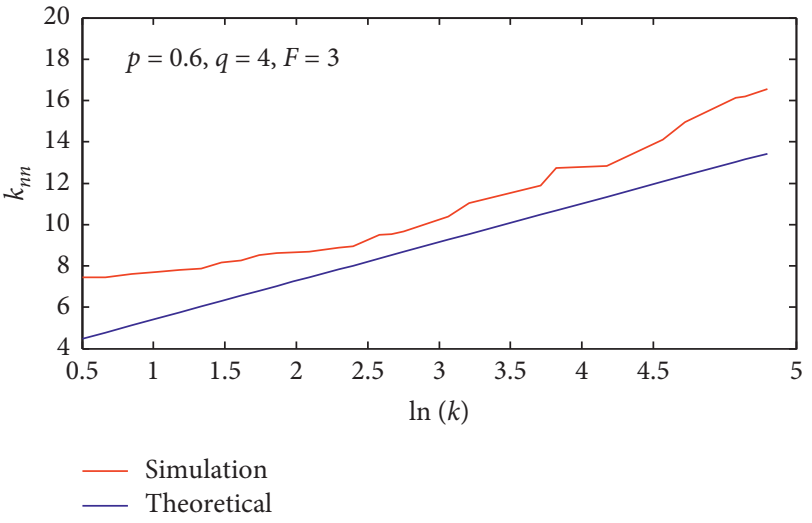

(b)

Figure 3: Comparison of simulated and theoretical mean degree of adjacent nodes at (a) $q=2$ and $F=3$ and (b) $q=4$ and $F=3$.

be seen that the actual simulation results were basically fit with theoretically derived results.

\section{Conclusions}

In this paper, we have considered the problem of assortativity in networks. Assortativity has a long history of study; however, little research investigated its formation by considering cultural traits of nodes. To fill the gap, this study established an assortative social network using two underlying mechanisms, i.e., preferential attachment and culture traits. Results indicated that the assortativity of a network was reduced due to the hubs formed by the preferential attachment mechanism. Such conclusion is in line with Newman [1]. Meanwhile, the higher the selection probability of culture trait-matching mechanism, the higher the assortativity of a network is. Hence, it is conclude that, to some extent, cultural similarity of nods formats assortativity in networks.

To further analyze the assortativity in detail, this study theoretically investigated the degree correlation of nodes. A conclusion was drawn that node degree presented a positive logarithmic correlation with the mean degree of adjacent nodes. Further, the actual simulation results were fitted with theoretically derived results. Therefore, it is considered that the introduction of a cultural trait-matching mechanism interpreted the formation mechanism of network assortativity. This study is valuable with regards to two aspects. On the one hand, our conclusions can be used to explain the origin of assortativity in social networks. Although it has been a common knowledge that preferential attachment is one of core mechanisms underlying building networks $[7,8]$, in the context of network assortativity, such mechanism functions negatively. Instead, cultural similarity forms the backbone of breeding assortativity. Hence, it is appropriate to interpret assortativity from a sociological perspective.

On the other hand, from the point view of cultural characteristics of nodes, the established network model effectively reflects the characteristics of real-life networks. The model is computationally efficient, with using the Monte Carlo simulation method, which puts applications to more social networks. The present study thus contributes to literature on assortativity by introducing a cultural traitmatching mechanism.

This study is not without its limitations. First, we only account for assortativity by degree of the nodes in social networks. Assortativity has, however, been applied to other topology-related characteristics of nodes as well, such as coreness, node betweenness, and node weight [6]. Second, we have here considered only the cultural traits of nodes, neglecting other sociology-related characteristics of nodes, e.g., race, gender, and age. Future research may generalize our method by taking account of more topology-related and 
sociology-related features of nodes. Third, we only investigate assortativity in the context of scale-free networks. The small average distance and large clustering coefficients of small-world networks also affect the formation of assortativity [12]. Future research could discuss this topic to extend the study of assortativity.

\section{Data Availability}

The data used to support the findings of this study are available from the corresponding author upon request.

\section{Conflicts of Interest}

The authors declare no conflicts of interest.

\section{Authors' Contributions}

Wei Wang, Xiaoming Sun, Yalan Wang, and Wentian Cui were responsible for the modeling and analysis. Wei Wang wrote the paper.

\section{Acknowledgments}

This study was supported by the National Natural Science Foundation of China (nos. 71402132 and 71472146) and Postdoctoral Foundation of China (no. 185622).

\section{References}

[1] M. E. J. Newman, “Assortative mixing in networks," Physical Review Letters, vol. 89, no. 20, p. 208701, 2002.

[2] C. R. Schwartz, "Trends and variation in assortative mating: causes and consequences," Annual Review of Sociology, vol. 39, no. 1, pp. 451-470, 2013.

[3] M. E. J. Newman, "Mixing patterns in networks," Physical Review E, vol. 67, no. 2, p. 026126, 2003.

[4] G. T. Cantwell and M. E. J. Newman, "Mixing patterns and individual differences in networks," Physical Review E, vol. 99, no. 4, p. 042306, 2019.

[5] M. McPherson, L. Smith-Lovin, and J. M. Cook, "Birds of a feather: homophily in social networks," Annual Review of Sociology, vol. 27, no. 1, pp. 415-444, 2001.

[6] R. Noldus and P. Van Mieghem, "Assortativity in complex networks," Journal of Complex Networks, vol. 3, no. 4, pp. 507-542, 2015.

[7] A.-L. Barabasi and R. Albert, "Emergence of scaling in random networks," Science, vol. 286, no. 5439, pp. 509-512, 1999.

[8] A. L. Barabasi, R. Albert, and H. Jeong, "Mean-field theory for scale-free random networks," Physica A: Statistical Mechanics and Its Applications, vol. 272, no. 1-2, pp. 173-187, 1999.

[9] M. E. J. Newman and J. Park, "Why social networks are different from other types of networks," Physical Review E, vol. 68, no. 3, p. 036122, 2003.

[10] M. Catanzaroa, G. Caldarelli, and L. Pietronero, "Social network growth with assortative mixing," Physica A: Statistical Mechanics and Its Applications, vol. 338, no. 1-2, pp. 119-124, 2004.

[11] M. Catanzaroa, G. Caldarelli, and L. Pietronero, "Assortative model for social networks," Physical Review E, vol. 70, no. 3, p. 037101, 2004.

[12] Q. Guo, T. Zhou, J.-G. Liu, W.-J. Bai, B.-H. Wang, and M. Zhao, "Growing scale-free small-world networks with tunable assortative coefficient," Physica A: Statistical Mechanics and Its Applications, vol. 371, no. 2, pp. 814-822, 2006.

[13] J. G. Liu, Y. Z. Dang, W. X. Wang et al., "Self-learning mutual selection model for weighted networks," 2005, https://arxiv. org/abs/physics/0512270.

[14] A. Vázquez, "Growing network with local rules: preferential attachment, clustering hierarchy, and degree correlations," Physical Review E, vol. 67, no. 5, p. 056104, 2003.

[15] R. Xulvi-Brunet and I. M. Sokolov, "Reshuffling scale-free networks: from random to assortative," Physical Review E, vol. 70, no. 6, p. 066102, 2004.

[16] P. Block and T. Grund, "Multidimensional homophily in friendship networks," Network Science, vol. 2, no. 2, pp. 189-212, 2014.

[17] L. Peel, J.-C. Delvenne, and R. Lambiotte, "Multiscale mixing patterns in networks," Proceedings of the National Academy of Sciences, vol. 115, no. 16, pp. 4057-4062, 2018.

[18] M. Piraveenan, M. Prokopenko, and A. Y. Zomaya, "Local assortativeness in scale-free networks," Europhysics Letters, vol. 89, no. 4, p. 28002, 2008.

[19] M. Piraveenan, M. Prokopenko, and A. Y. Zomaya, "On congruity of nodes and assortative information content in complex networks," Networks and Heterogeneous Media, vol. 7, no. 3, pp. 441-461, 2012.

[20] K. M. Altenburger and J. Ugander, "Monophily in social networks introduces similarity among friends-of-friends," Nature Human Behaviour, vol. 2, no. 4, pp. 284-290, 2018.

[21] A. P. Quayle, A. S. Siddiqui, and S. J. M. Jones, "Modeling network growth with assortative mixing," The European Physical Journal B, vol. 50, no. 4, pp. 617-630, 2006.

[22] C. Nagoshi, R. Johnson, and G. Danko, "Assortative mating for cultural identification as indicated by language use," Behaviour Genetics, vol. 20, no. 1, pp. 23-31, 1990.

[23] J. Moody, "Race, school integration, and friendship segregation in America," American Journal of Sociology, vol. 107, no. 3, pp. 679-716, 2001.

[24] J. Ugander, B. Karrer, L. Backstrom, and C. Marlow, "The anatomy of the facebook social graph," 2011, https://arxiv.org/ abs/1111.4503.

[25] T. Postmes, S. A. Haslam, and R. I. Swaab, "Social influence in small groups: an interactive model of social identity formation," European Review of Social Psychology, vol. 16, no. 1, pp. 1-42, 2005.

[26] D. Krackhardt and M. Kilduff, "Structure, culture and Simmelian ties in entrepreneurial firms," Social Networks, vol. 24, no. 3, pp. 279-290, 2002.

[27] R. Axelrod, “The dissemination of culture," Journal of Conflict Resolution, vol. 41, no. 2, pp. 203-226, 1997.

[28] D. Centola, J. C. González-Avella, V. M. Eguíluz, and M. San Miguel, "Homophily, cultural drift, and the co-evolution of cultural groups," Journal of Conflict Resolution, vol. 51, no. 6, pp. 905-929, 2007.

[29] A. Galeotti, S. Goyal, and J. Kamphorst, "Network formation with heterogeneous players," Games and Economic Behavior, vol. 54, no. 2, pp. 353-372, 2006.

[30] H. Haller and S. Sarangi, "Nash networks with heterogeneous links," Mathematical Social Sciences, vol. 50, no. 2, pp. 181-201, 2005.

[31] R. Albert and A. L. Barabási, "Statistical mechanics of complex networks," Reviews of Modern Physics, vol. 74, no. 1, pp. 47-97, 2002.

[32] X. H. Xiao, G. W. Ye, B. Wang, and M. F. He, "Cultural dissemination in a complex network," Physica A: Statistical 
Mechanics and Its Applications, vol. 388, no. 5, pp. 775-779, 2009.

[33] K. Klemm, V. M. Eguiluz, R. Toral, and M. San Miguel, "Globalization, polarization and cultural drift," Journal of Economic Dynamics \& Control, vol. 29, no. 1-2, pp. 321-334, 2005.

[34] A. Barrat and R. Pastor-Satorras, "Rate equation approach for correlations in growing network models," Physical Review E, vol. 71, no. 3, p. 036127, 2005. 